\title{
Multilocular pyogenic liver abscess with poorly liquefied content: a potential therapeutic concern
}

\begin{abstract}
Background: Percutaneous catheter drainage (PCD) is currently the preferred therapeutic procedure of pyogenic liver abscesses (PLAs). However, multilocular poorly liquefied PLAs (MPLPs) are unsuitable for PCD. As reports on MPLPs are scarce, we aimed to explore possible therapeutic strategies for this serious type of PLA.

Methods: The medical records of patients with PLA who were hospitalized at our institution between January 2011 to December 2015 were retrospectively reviewed. Demographic factors, biochemical, bacteriological, and imaging results, and treatment outcomes were evaluated.

Results: Fourteen patients with MPLPs and 37 with drainable PLAs were identified and included in the study. Clinical characteristics were generally similar between the two groups. Klebsiella pneumoniae accounted for the majority of the culprit pathogens in MPLPs. Patients with MPLPs were managed medically without PCD. Despite this, treatment outcomes were not inferior compared to patients with drainable PLAs. Contrast-enhanced images revealed that the intricate septa of the MPLP maintained its blood perfusion. Most MPLPs did not liquefy over the treatment period.

Conclusion: We should remember that MPLP is not rare. The septa may have delivered antibiotics adequately into the MPLPs, thus producing good treatment outcomes. Despite our findings, a high level of suspicion of treatment failure is crucial in these patients to ensure favorable treatment outcomes.
\end{abstract}

Keywords: pyogenic liver abscess, multilocular, liquefaction, drainage, klebsiella pneumonia
Volume 8 Issue 3 - 2017

\author{
Kazuhiko Morii,' Takeharu Yamamoto,' \\ Shinichiro Nakamura,' Shigeyoshi Fujiwara, ${ }^{2}$ \\ Hiroaki Okushin' \\ 'Department of Hematology, Japanese Red Cross Society Himeji \\ Hospital, Japan \\ ${ }^{2}$ Department of Oral and Maxillofacial Surgery, Japanese Red \\ Cross Society Himeji Hospital, Japan
}

Correspondence: Kazuhiko Morii, Department of Hepatology, Japanese Red Cross Society Himeji Hospital, I- I2-I Shimoteno, Himeji, Hyogo 670-8540, Japan, Tel +8I 79-294-225I, Fax +8I 79-296-4050, Email moriikazuhiko@gmail.com

Received: November 08, 2017 | Published: December 28 2017
Abbreviations: ESBL, extended-spectrum $\beta$-lactamase; PCD, percutaneous catheter drainage; PLA, pyogenic liver abscess; MPLP, multilocular poorly liquefied pla

\section{Introduction}

Pyogenic liver abscess (PLA) is the most frequent, potentially life-threatening type of visceral abscess, with an increasing incidence worldwide. ${ }^{1,2}$ The etiologies and microbiological spectrum show distinct regional differences around the world. Klebsiella pneumoniae is the most common causative pathogen of PLA in eastern Asian countries, ${ }^{3-5}$ whereas Streptococcus spp. predominates in Europe, Canada, and Australia. ${ }^{6-10}$ However, recently, Klebsiella pneumoniae has become a more frequent causative pathogen of PLA in Western countries. ${ }^{11,12}$

Although there is no consensus on the guidelines regarding optimal management strategies of PLA, percutaneous catheter drainage (PCD) is currently the initial treatment option of choice. ${ }^{13,14}$ In the majority of patients, PCD is reported to be a safe and sufficient treatment, even for huge PLAs measuring $\geq 10 \mathrm{~cm} .{ }^{15}$ In this modern era of minimally invasive PLA management, multilocular poorly liquefied PLA (MPLP) remains a challenge. MPLP is unsuitable for PCD due to its intricate septations and thick viscid pus, and therefore it is treated rather reluctantly with antibiotics alone. This conservative treatment strategy sometimes raises concerns about the risk of treatment failure and certain complications. Indeed, we have experienced a case of huge MPLP with an intraperitoneal rupture who required surgical treatment. ${ }^{16}$ This MPLP was caused by Klebsiella pneumoniae infection, which is reported to be often associated with multilocular PLA. ${ }^{17,18}$
Diagnosis of multiloculation of PLA may vary depending on the capacity of the imaging modalities and the reader's subjective interpretation. According to the previous studies, multilocular abscesses account for about $50 \%$ of PLAs. ${ }^{15,19,20}$ Half of these multilocular PLAs were difficult to be managed with PCD in our recent experience, and accordingly, MPLPs may account for approximately a quarter of PLAs (Figure 1). Despite the relatively high prevalence, there are few reports on MPLPs that describe their clinical characteristics and treatment outcomes.

Therefore, this study was aimed to investigate MPLPs, comparing their clinical and therapeutic characteristics with those of liquefied drainable PLAs. Further, in this study, we aimed to elucidate the unique abscess structures, clinical and bacteriological features, and treatment outcomes for MPLPs.

\section{Patients and methods}

\section{Study design}

The present study is a retrospective cohort analysis of patients with PLA who were hospitalized at our institute between January 2011 and December 2015. Our institute is a community-based tertiary referral hospital with a specialized hepatology department. The patient inclusion criteria were as follows: (a) patients who were hospitalized at our institute because of PLA between January 2011 and December 2015, and (b) the primary reason for hospitalization was a PLA. The exclusion criterion was the coexistence of presumable primary infectious foci other than PLAs. Electronic medical records were reviewed to evaluate clinical presentation, demographic features, biochemical and serological markers, imaging findings, microbiology 
of pathogenic organisms, antimicrobial resistance testing, and the therapeutic course of the illness. The ethics committees of our institutional review board approved this study in accordance with the Declaration of Helsinki (number CTM16-011).

\section{Diagnosis of PLA}

The initial workup involved abdominal ultrasonography, and if PLA was suspected, computed tomography (CT) was performed to define its anatomical features in detail and to determine its route of infection. In case of biliary-origin PLA, magnetic resonance cholangiopancreatography was used to investigate biliary tract pathologies. Diagnosis of PLA was based on the following criteria: (a) the clinical signs of bacterial infection such as fevers with leukocytosis, (b) the characteristic imaging features with ultrasonography (US) and computed tomography (CT), (c) samples consistent with PLAs were obtained by image-guided aspiration, (d) positive bacterial cultures of blood or pus, (e) resolution of the lesions with antibiotic therapy with/without drainages, (f) a negative fluorescent antibody test against Entamoeba histolytica, and (g) surgical findings, if available, were consistent with PLAs. ${ }^{2}$

The characteristic imaging features of PLAs were regarded as follows: (a) solitary or multiple lesions tending to coalesce focally, (b) usually well-defined hypoattenuating lesions with faint rim enhancement and perilesional edema by contrast-enhanced CT, (c) unilocular with smooth margins or multilocular with septations and an irregular contour, (d) varying degrees of internal contents depending on the stage of suppuration, liquefaction, and fibrosis, and (e) occasionally containing gas in the lesions. ${ }^{21}$

Multilocular abscess was defined as an abscess with 2 or more septa. MPLP was defined as a multilocular abscess in which proper source control could not be achieved by PCD due to complex septations and poorly liquefied contents (Figure 1). If a multilocular PLA had liquefied contents and could be effectively managed with PCD, it was categorized as a drainable PLA (a multilocular drainable PLA, Figure 2).
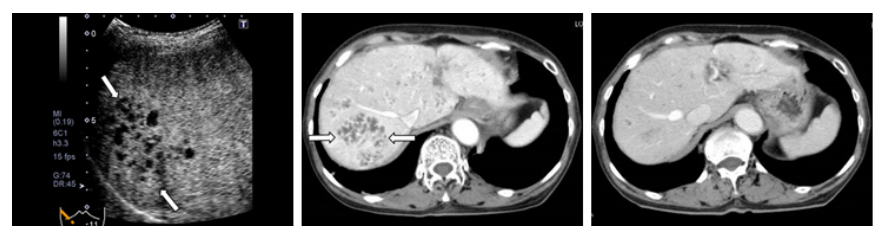

Figure I Images of a 76-year-old woman showing a number of MPLPs associated with Klebsiella pneumonia.

A) The post-vascular phase of perfluorobutane-enhanced ultrasonography. B) Contrast-enhanced CT scan. Both (A) and (B) show images obtained before treatment. These images demonstrate the enhancing intricate septation of the MPLP, which exhibits a honeycomb-pattern (arrows).C) Contrast-enhanced CT scan obtained 4 weeks after the antibiotic introduction shows marked improvement of the MPLPs.

MPLP denotes multilocular poorly liquefied pyogenic liver abscess.

\section{Data collection}

The following variables were explored: sex, age, presenting symptoms, interval from symptomatic onset to diagnosis, route or etiology of infection, preexisting comorbidities, length of hospital stay, and treatment outcomes at discharge (recovery or deceased). Routes of infection were divided into biliary-, portal pyemia-, urinary tract infection-, hematogenous-, and cryptogenic-origin. Biliaryorigin routes included cholecystitis, cholangitis, and pancreatitis. Portal pyemia-origin routes implied ascending portal venous sepsis and included clinical presentations such as colonic diverticulitis, enteritis, appendicitis, inflammatory bowel disease, and supra-infected gastrointestinal neoplasms (i.e. ulcerated tumors that allowed bacterial invasion). Hematogenous-origin routes were defined as secondary to hematogenous seeding with a documented bacteremia before the onset of PLA and included clinical pictures such as skin and soft tissue infection and catheter-related blood stream infection. Cryptogenicorigin routes were defined as having no identifiable source of infection despite extensive explorations. Preexisting comorbidities included diabetes mellitus, alcoholic abuse, prior gastroduodenal ulcer, prior abdominal surgery, cardiovascular diseases (hypertension, ischemic heart disease, stroke), and chronic renal failure, all of which have been reported to be associated with PLA fatality. ${ }^{1,2,22,23}$

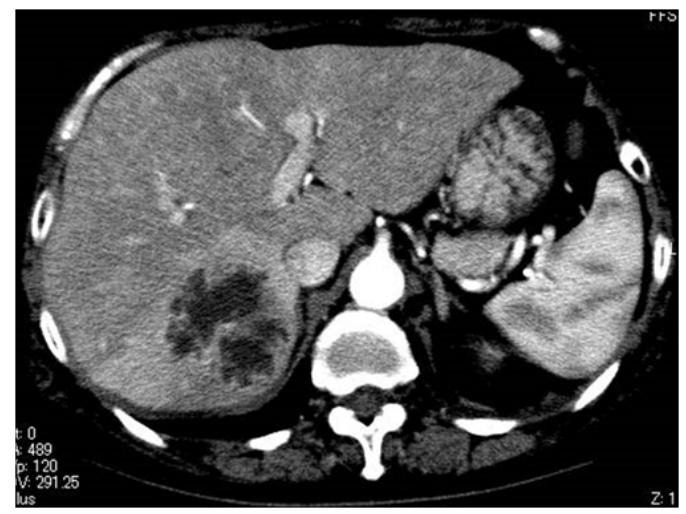

Figure 2 Images of multilocular drainable PLAs.

Contrast-enhanced CT scan of a 70-year-old woman shows a multilocular PLA associated with Klebsiella pneumoniae. This abscess had been effectively managed with percutaneous drainage by breaking through the septa with the catheter. This is not categorized as MPLPs but drainable PLAs.

PLA denotes pyogenic liver abscess.

Imaging findings were recorded including the number of distinct abscess cavities, their lobar distribution in the liver, their diameters, whether the contents of the PLAs were uni- or multilocular and liquefied or poorly liquefied, and gas-formation. If there were multiple lesions, the largest diameter was recorded. We investigated whether liquefaction of the MPLPs was observed over the treatment courses. All laboratory tests, blood culture samplings, and imaging studies were performed at the initial assessment. All pus specimens were sent for culture during PCD or surgery. Blood and pus samples were obtained for aerobic and anaerobic cultures. Extended-spectrum $\beta$-lactamase (ESBL) production was examined when strains of Klebsiella spp. and Escherichia coli were isolated.

\section{Principal treatment strategies for PLA}

Treatment procedures consisted of medical treatment, imageguided PCD, and surgical intervention. After the referral of patients with presumable PLA, blood cultures were performed, and empirical treatments with broad-spectrum antibiotics were commenced immediately. A commonly used empirical antibiotic is the thirdgeneration cephalosporin (e.g. ceftriaxone). The antibiotic treatment was optimized afterward according to the results of the bacterial cultures and antimicrobial susceptibility analysis of the isolated pathogens. Patients with abscesses larger than $3 \mathrm{~cm}$ in diameter predominantly underwent source control by PCD with 7 Fr catheter drainage within 24hours, except in the following situations: (a) when the lesions were MPLPs, and (b) when there was a safety concern about PCD such as bleeding tendencies and a moderate-high amount of ascites. ${ }^{13,14,24}$ The duration of drainage catheter placement ranged 
from 4 to 10days. The methodological contrivances of PCD for multilocular PLAs were as follows: (a) breaking through the septa with catheter manipulation to make separated cavities contiguous (Figure 2), and (b) placing a drainage catheter in the main lumen if a PLA had a large main lumen with several ancillary lumens.

Endoscopic biliopancreatic drainage was performed when necessary. Surgical intervention was the last treatment option when medical treatment and PCD failed ${ }^{23}$ and was only required for one patient with a ruptured abscess. ${ }^{16}$ Finally, the resolution of the PLA was confirmed by follow-up imaging studies.

\section{Statistical analysis}

The primary statistical objective was to identify meaningful differences in clinical characteristics or laboratory data between patients with MPLPs and drainable PLAs. Standard statistical methods were used to analyze the results. Continuous variables were compared using the Mann-Whitney rank-sum or Student's T test and categorical variables by chi-square tests. Continuous variables are presented as mean \pm standard deviation or median and categorical variables as absolute values or percentages. P values below 0.05 (two-tailed) were considered statistically significant. All analyses were performed using the EZR v1.26 software program. ${ }^{25}$

\section{Results}

After application of the inclusion and exclusion criteria, a total of 51 patients were included in this study (Table 1). Twenty-six (51\%) patients had multilocular PLAs and the other 25 (49\%) unilocular PLAs. Twelve of the 26 patients with multilocular PLA could be effectively managed with PCD, and accordingly the remaining $14(27 \%)$ patients accounted for those with MPLPs. Patients with drainable PLAs consisted of 12 patients with multilocular PLAs and all of 25 with unilocular PLAs. The baseline demographic and clinical characteristics of the patients with MPLP and drainable PLA were very similar (Table $1 \& 2$ ). The chief complaint, abdominal pain, tended to be less common in patients with MPLP. The location, number, and diameters of the abscesses were not different between the two groups. MPLPs were significantly associated with a cryptogenic source of infection.

Laboratory data were also not different between the two groups (Table 3). The overall positive rate of bacterial culture was 51\%. The isolated bacterial strains had good sensitivity to commonly used antibiotics, and no ESBL-producing strains were found. Seven of the 14 patients with MPLPs showed positive bacterial culture results: $6(86 \%)$ of them were Klebsiella pneumoniae, and only $1(14 \%)$ was Streptococcus bovis. Conversely, Klebsiella pneumoniae was isolated in 14 patients, $6(43 \%)$ of whom had MPLP. Thus, patients with MPLPs were significantly more likely to exhibit Klebsiella pneumoniae infection than those with drainable PLAs. In addition, 7 of 12 patients with multilocular drainable PLAs exhibited positive bacterial culture results: 4 (57\%) of them were Klebsiella pneumoniae.

Twenty-seven $(53 \%)$ patients were treated with antibiotic treatment alone, and the other $24(47 \%)$ patients with a combination of antibiotics and PCD (Table 2). Although all patients with MPLPs were treated without $\mathrm{PCD}$, this conservative approach was successful in $92 \%$ (13 of 14) of patients. Treatment outcomes, e.g. length of hospital stay, were similar in the MPLP and drainable PLA groups (Table 2). Only $1(2 \%)$ patient with a huge MPLP required conversion to surgical treatment because of intra-abdominal abscess rupture. ${ }^{16}$ Forty-nine (96\%) patients were discharged alive with resolution of the abscesses. There were $2(4 \%)$ fatalities, and both were attributed to septic multiorgan failure. Fatality rates were not different for the MPLP and drainable PLA groups. Liquefaction of the abscess contents was not observed in all but 1 MPLPs over the ensuing weeks (Figure $3)$. These abscesses shrunk in response to antibiotic administration, and their cavities were gradually replaced by fibrous granulation.

$†$ MPLP denotes multilocular poorly liquefied pyogenic liver abscess, $\$$ PLA pyogenic liver abscess.

†INR denotes international normalized ratio.

Table I Clinical and biochemical variables of individuals with overweight-obesity

\begin{tabular}{|c|c|c|c|}
\hline & MPLP & Drainable & PValue \\
\hline Patients, $\mathrm{n}$ (male: female) & $14(||: 3)$ & $37(24: 13)$ & 0.3 \\
\hline Age, year & $68.8 \pm 13.1$ & $72.2 \pm 12.2$ & 0.5 \\
\hline Symptomatic onset to diagnosis, days & $6.4 \pm 7.6$ & $6.6 \pm 6.4$ & 0.5 \\
\hline \multicolumn{4}{|l|}{ Clinical presentation, n (\%) } \\
\hline Fever and chills & $10(7 \mid .4)$ & $21(56.7)$ & 0.3 \\
\hline Abdominal pain & I (7.I) & $10(27.0)$ & 0.1 \\
\hline General malaise & $3(21.4)$ & $4(10.8)$ & 0.3 \\
\hline Anorexia & 0 & $2(5.4)$ & 0.4 \\
\hline Number of abscess cavities, $\mathrm{n}$ & $2.7 \pm 1.9$ & $2.0 \pm 2.0$ & 0.6 \\
\hline range, n (\%) I & $10(7 \mid .4)$ & $26(70.3)$ & \\
\hline $2-4$ & $3(2 \mid .4)$ & $5(13.5)$ & \\
\hline$\geq 5$ & I (7.I) & $6(16.2)$ & \\
\hline \multicolumn{4}{|l|}{ Location of abscess, $\mathrm{n}(\%)$} \\
\hline Right lobe & $10(7 \mid .4)$ & $25(67.6)$ & 0.8 \\
\hline Left lobe & $2(14.3)$ & $9(24.3)$ & 0.4 \\
\hline Bilateral lobes & $2(14.3)$ & $3(8.1)$ & 0.5 \\
\hline Diameter of the abscess, $\mathrm{cm}$ & $5.9 \pm 2.5$ & $6.0 \pm 3.2$ & 0.6 \\
\hline range, $\mathrm{n}(\%) \leq 3 \mathrm{~cm}$ & $3(2 \mathrm{I} .4)$ & $10(27.0)$ & \\
\hline $3.1-5.0 \mathrm{~cm}$ & $4(28.6)$ & $5(13.5)^{\prime}$ & \\
\hline $5.1-7.4 \mathrm{~cm}$ & $4(28.6)$ & II (29.7) & \\
\hline $7.5-9.9 \mathrm{~cm}$ & 0 & $7(18.9)$ & \\
\hline$\geq 10 \mathrm{~cm}$ & $3(2 \mid .4)$ & $4(10.8)$ & \\
\hline \multicolumn{4}{|l|}{ Route of infection, $\mathrm{n}(\%)$} \\
\hline Cryptogenic & $10(7 \mid .4)$ & $10(27.0)$ & 0.003 \\
\hline Biliary & $3(2 \mid .4)$ & $16(43.2)$ & 0.1 \\
\hline Portal pyemia & I (7.I) & $7(18.9)^{\prime}$ & 0.3 \\
\hline Hematogenous & 0 & $2(5.4)$ & 0.4 \\
\hline Urinary tract infection & 0 & $2(5.4)$ & 0.4 \\
\hline
\end{tabular}


Table 2 Underlying conditions and treatment outcomes in patients with MPLPs and drainable PLAs

\begin{tabular}{|c|c|c|c|}
\hline & MPLP & Drainable & $P$ value \\
\hline Underlying diseases, n (\%) & 14 & 37 & \\
\hline Diabetes mellitus & $6(42.9)$ & $14(37.8)$ & 0.7 \\
\hline Biliary disease & $2(14.3)$ & $12(32.4)$ & 0.2 \\
\hline Pancreatic disease & $\mathrm{I}(7.1)$ & $2(5.4)$ & 0.8 \\
\hline Liver cirrhosis & I (7.I) & $4(10.8)$ & 0.7 \\
\hline Gastroduodenal ulcer & 0 & $4(10.8)$ & 0.2 \\
\hline Inflammatory bowel disease & 0 & $2(5.4)$ & 0.4 \\
\hline Colonic diverticulitis & 0 & $3(8.1)$ & 0.3 \\
\hline Hypertension & $3(2 \mid .4)$ & II (29.7) & 0.6 \\
\hline Ischemic heart disease & $2(14.3)$ & $2(5.4)$ & 0.3 \\
\hline Stroke & 0 & I (2.7) & 0.5 \\
\hline Chronic renal failure & $3(21.4)$ & $2(5.4)$ & 0.08 \\
\hline \multicolumn{4}{|l|}{ Risk factors, n (\%) } \\
\hline Smoking & $3(21.4)$ & $8(2 \mid .6)$ & 0.9 \\
\hline Prior abdominal surgery & $4(28.6)$ & $4(10.8)$ & 0.1 \\
\hline Alcoholic abuse & $3(21.4)$ & $5(13.5)$ & 0.5 \\
\hline Prior malignancy & I (7.I) & $4(10.8)$ & 0.7 \\
\hline Immunosuppressive medication & I (7.I) & $2(5.4)$ & 0.8 \\
\hline Percutaneous catheter drainage & 0 & 24 & - \\
\hline Surgical treatment & I & 0 & 0.5 \\
\hline Length of hospital stay, days & $24.4 \pm 16.2$ & $20.3 \pm 14.6$ & 0.3 \\
\hline Fatality, n (\%) & I (7.I) & I (2.7) & 0.5 \\
\hline
\end{tabular}

Table 3 Laboratory and bacteriological test results in patients with MPLPs and drainable PLAs

\begin{tabular}{|c|c|c|c|c|}
\hline & Reference Range & MPLP & Drainable & PValue \\
\hline Peripheral white-cell count, /mm3 & $3,300-8,600$ & $\mathrm{II}, 200 \pm 4,000$ & $10,100 \pm 4,700$ & 0.5 \\
\hline Total bilirubin, mg/dL & $0.4-1.5$ & $1.6 \pm 1.5$ & $1.5 \pm 1.9$ & 0.3 \\
\hline Albumin, $g / d L$ & 4. $1-5.1$ & $2.9 \pm 0.8$ & $3.1 \pm 0.5$ & 0.8 \\
\hline Prothrombin-time INR & & $1.2 \pm 0.2$ & $1.2 \pm 0.3$ & 0.7 \\
\hline Alanine aminotransferase, $U / L$ & $7-23$ & $92 \pm 147$ & $|64 \pm 53|$ & I \\
\hline Alkaline phosphatase, $U / L$ & $106-322$ & $505 \pm 257$ & $609 \pm 373$ & 0.5 \\
\hline Creatinine, $\mathrm{mg} / \mathrm{dL}$ & $0.46-0.79$ & $1.0 \pm 0.4$ & $0.9 \pm 0.4$ & 0.6 \\
\hline C-reactive protein, $\mathrm{mg} / \mathrm{L}$ & $0-1.4$ & $|50 \pm 8|$ & $150 \pm 97$ & 0.9 \\
\hline Procalcitonin, ng/mL & $\leq 0.05$ & $8.3 \pm 11.7$ & $6.1 \pm 13$ & 0.9 \\
\hline Overall culture positive rate, $\mathrm{n}(\%)$ & & $7(50)$ & $19(51.3)$ & 0.9 \\
\hline Positive blood culture, n (\%) & & $7(50)$ & $12(32.4)$ & 0.2 \\
\hline Positive pus culture, $\mathrm{n}(\%)$ & & - & $17(45.9)$ & - \\
\hline \multicolumn{5}{|l|}{ Isolated bacterial pathogens, $\%$} \\
\hline Klebsiella pneumoniae & & 42.9 & 21.6 & 0.004 \\
\hline Streptococcus anginosus group & & 0 & 10.8 & $<0.001$ \\
\hline Enterococcus spp. & & 0 & 8.1 & 0.003 \\
\hline Escherichia coli & & 0 & 5.4 & 0.02 \\
\hline others & & 7.1 & 5.4 & 0.5 \\
\hline negative culture & & 50.0 & 48.7 & 0.9 \\
\hline
\end{tabular}
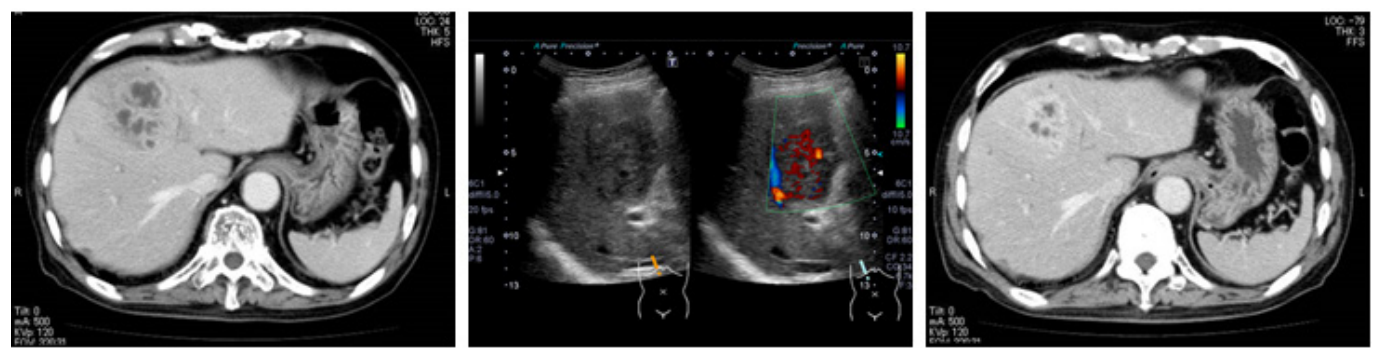

Figure 3 Images of a 75-year-old man showing MPLP changes associated with Klebsiella pneumonia.

A) The image shows contrast-enhanced CT scan, obtained before treatment. The CT scan demonstrates a multilocular abscess with enhancing rim and septa. This finding indicates that the internal septa are maintaining their blood perfusions.

B) Ultrasonography obtained before treatment shows a poorly-liquefied abscess with color Doppler signals in the internal septa, indicating that the septa are maintaining their blood perfusions.

C) A week after the treatment, contrast-enhanced CT scan shows the abscess has decreased in size without lequefaction of the contents.

\section{Discussion}

This study aimed to evaluate MPLPs in terms of clinical and therapeutic characteristics, in comparison with drainable PLAs. Fourteen (27\%) patients of our cohort had MPLPs. Patients with MPLPs had significantly higher incidences of Klebsiella pneumoniae infection and cryptogenic source of infection. These findings are in line with prior reports that Klebsiella pneumoniaeassociated PLA is often cryptogenic and commonly presents as a single large abscess with predominantly multilocular content. ${ }^{17,18}$ One 
of the reasons that Klebsiella pneumoniae infection is often associated with MPLP is speculated as follows. A loss or reduction of capsule synthesis by the pathogenic Klebsiella pneumoniae strain renders an antiphagocytic effect against macrophages and neutrophils, ${ }^{26}$ and this predisposes the pathogen to invade and destroy the parenchyma rapidly, without allowing enough time for the tissues to break down and liquefy into homogeneous pus. ${ }^{27}$ These MPLAs were unsuitable for PCD and often managed with antibiotic administration alone. We initially assumed that a MPLP reflected its immature stage and might subsequently shift to a liquefaction process that would make PCD available. However, most abscesses did not liquefy, but simply shrank over the following treatment weeks (Figure 3). The reason why conservative treatment without PCD was generally successful in our cohort with MPLPs can be explained as follows: the internal septa of the MPLPs extended intricately into the abscess cavities, maintaining their blood perfusion, and therefore allowing the antibiotics to penetrate adequately into the abscess (Figures $1 \& 3$ ).

Multilocular PLAs larger than $3 \mathrm{~cm}$ have been previously recommended to be managed with surgical intervention. ${ }^{13}$ In addition, greater age ( $\geq 55$ years), presence of multiple abscesses, malignancy as etiology, and endoscopic intervention were reported to be the risk factors for the development of PLA treatment failure with antibiotics alone. ${ }^{24}$ In our cohort, $50 \%$ of MPLPs were larger than $5 \mathrm{~cm}$ in diameter and $29 \%$ were multiple. Despite these unfavorable factors, the overall fatality rate in our cohort was $4 \%$, which was equal to or better than rates reported in previous studies about PLAs (Table 2). ${ }^{1,2,22,23,28}$ Fatality rates did not differ between MPLP and drainable PLA groups in our cohort. The new knowledges from this study are: (i) about a quarter of PLAs may be MPLPs, i.e. unsuitable for PCD, (ii) MPLP often results from Klebsiella pneumoniae infection, (iii) the intricate septa of MPLP may maintain their blood flow, (iv) most such MPLPs shrink to disappear without liquefaction following antibiotic treatment, and (v) importantly, the conservative treatment outcomes of MPLPs may not be associated with inferior outcomes.

How to apply these knowledges for routine clinical practice? We recommend: (a) in case of MPLP unsuitable for PCD, it should be carefully examined whether the septa maintain their blood perfusions with imaging studies, (b) the adequate antibiotic penetration into the abscess is essential for a successful conservative treatment, and (c) MPLP that has septa with well-preserved blood flow can often be managed medically without PCD. Of course, prompt decision to proceed with more aggressive management is mandatory in emergency situations. Alkofer et al., ${ }^{23}$ determined two situations that lead to surgical PLA management. ${ }^{23}$ The first situation involves factors that increase the risk of PCD failure: multilocular abscesses, gas-forming abscesses, and septic shock at presentation. The second situation involves factors associated with high mortality: poor physiological state, severe sepsis, pleural effusion, and a malignant PLA origin. Unfortunately, there are few salvage options except for surgical treatment, when medical treatment of MPLP fails. ${ }^{29} \mathrm{Ke}$ et al., ${ }^{30}$ suggested preliminary radiofrequency ablation as an alternative to surgical intervention in patients unsuitable for $\mathrm{PCD}^{30}$. Kusumoto et al., ${ }^{31}$ reported a continuous perfusion drainage procedure in which two tubes were placed to accelerate irrigation and excretion of the abscess contents, respectively. ${ }^{31}$

Two recent literatures about PLA from China reported that PLA patients treated without PCD were $19.6 \%$ and $10.6 \%$. Thirty three percent and all of them were surgically drained, respectively. ${ }^{32,33}$ They also alerted that about $10 \%$ of the patients with Klebsiella pneumoniae-associated PLA had invasive liver abscess syndrome with distant metastasis infection, as compared to only one $(0.2 \%)$ of our cohort. ${ }^{16}$ On the other hand, a report from Spain revealed that $31.6 \%$ of patients with PLA were treated with antibiotics alone. ${ }^{34}$ Unfortunately, these literatures do not mention the loculation of PLAs. There may be geographical difference in the prevailing culprit bacterial strains and their virulence.

This study had several limitations. First, the sample size was small, and this may detract from the statistical strength of our findings. Second, this was a retrospective study based on medical records from routine clinical practice. This limited the ability to provide exhaustive information. Third, our patient group may exhibit regional variations that are not reflected in the general population. Thus, our findings should be validated in a larger population.

\section{Conclusion}

In our retrospective cohort analysis of 51 consecutive patients with PLA, $14(27 \%)$ patients had MPLPs, and thus, we need to remember that MPLP is not rare. Klebsiella pneumoniae accounted for the majority of identified pathogens in these patients. MPLPs were managed with antibiotics alone. Despite this conservative treatment strategy, the length of hospital stays and fatality rates of the patients with MPLPs was not inferior to that of the patients with drainable PLAs. The intricate septa of the MPLP maintains its blood perfusion and may have delivered antibiotics adequately into the MPLPs, thus producing good treatment outcomes. Nevertheless, high suspicion of treatment failure is crucial in patients with MPLPs to ensure that management strategies are implemented in a timely manner.

\section{Conflicts of Interest}

The authors declare that they have no conflict of interest (COI).

\section{Acknowledgements}

We would like to acknowledge the staffs in our Hepatology Department for their assistance: Mitsuki Sumino, Hidemi Nakaida, Rina Nishikawa, Kana Umei, Ayumi Irie, Misaki Higashihata, Ryota Ohshima, and Kayo Sekiguchi.

\section{Funding}

None.

\section{References}

1. Meddings L, Myers RP, Hubbard J, et al. A population-based study of pyogenic liver abscesses in the United States: incidence, mortality, and temporal trends. Am J Gastroenterol. 2010;105(1):117-124.

2. Tian LT, Yao K, Zhang XY, et al. Pyogenic liver abscesses in adult patients with and without diabetes mellitus: an analysis of the clinical characteristics, features of the causative pathogens, outcomes and predictors of fatality: a report based on a large population, retrospective study in China. Clin Microbiol Infect. 2012;18(9):E314-330.

3. Yang $\mathrm{CC}$, Yen $\mathrm{CH}$, Ho MW, et al.Comparison of pyogenic liver abscess caused by non-Klebsiella pneumoniae and Klebsiella pneumoniae. $J$ Microbiol Immunol Infect. 2004;37(3):176-184.

4. Okano H, Shiraki K, Inoue H, et al. Clinicopathological analysis of liver abscess in Japan. Int J Mol Med. 2002;10(5): 627-630

5. Luo M, Yang XX, Tan B, et al. Distribution of common pathogens in patients with pyogenic liver abscess in China: a meta-analysis. Eur $J$ Clin Microbiol Infect Dis. 2016;35(10):1557-1565.

6. Mohsen AH, Green ST, Read RC, et al.Liver abscess in adults: ten years' experience in a UK centre. QJM . 2002;95(12):797-802. 
7. Cerwenka H. Pyogenic liver abscess: Differences in etiology and treatment in Southeast Asia and Central Europe. World J Gastroenterol. 2010;16(20):2458-2462.

8. Kaplan GG, Gregson DB, Laupland KB. Population-based study of the epidemiology of and the risk factors for pyogenic liver abscess. Clin Gastroenterol Hepatol. 2004;2(11):1032-1038.

9. Hernandez RJJ, Mazorra LM, Martel CA, et al. Pyogenic liver abscesses: mortality-related factors. Eur J Gastroenterol Hepatol. 2007;19(10):853-858.

10. Pang TCY, Fung T, Samra J, et al.Pyogenic liver abscess: An audit of 10 years' experience. World J Gastroenterol. 2011;17(12):1622-1630.

11. Lederman ER, Crum NF. Pyogenic liver abscess with a focus on Klebsiella pneumonia as a primary pathogen: an emerging disease with unique clinical characteristics. Am J Gastroenterol. 2005;100(2):322-331.

12. Sobirk SK, Struve C, Jacobsson SG. Primary Klebsiella pneumoniae Liver Abscess with Metastatic Spread to Lung and Eye, a North-European Case Report of an Emerging Syndrome. Open Microbiol J. 2010;4:5-7.

13. Hope WW, Vrochides DV, Newcomb WL, et al.Optimal treatment of hepatic abscess. Am Surg. 2008;74(2):178-182.

14. Dietrich CF, Lorentzen T, Appelbaum L, et al. EFSUMB Guidelines on Interventional Ultrasound (INVUS), Part III - Abdominal Treatment Procedures (Long Version). Ultraschall Med. 2016;37(1):E1-32.

15. Ahmed S, Chia CL, Junnarkar SP, et al.Percutaneous drainage for giant pyogenic liver abscess-is it safe and sufficient? Am J Surg. 2016;211(1):95-101.

16. Morii K, Kashihara A, Miura S, et al. Successful hepatectomy for intraperitoneal rupture of pyogenic liver abscess caused by Klebsiella pneumoniae. Clin J Gastroenterol. 2012;(2)5:136-140.

17. Wang JH, Liu YC, Lee SS, et al. Primary liver abscess due to Klebsiella pneumoniae in Taiwan. Clin Infect Dis. 1998;26(6):1434-1438.

18. Hui JY, Yang MK, Cho DH, et al. Pyogenic liver abscesses caused by Klebsiellapneumoniae: US appearance and aspiration findings. Radiology. 2007;242(3):769-776.

19. Bernardino ME, Berkman WA, Plemmons M, et al. Percutaneous drainage of multiseptated hepatic abscess. J Comput Assist Tomogr. 1984;8(1):38-41.

20. Liu CH, Gervais DA, Hahn PF, et al.Percutaneous hepatic abscess drainage: do multiple abscesses or multiloculated abscesses preclude drainage or affect outcome? J Vasc Interv Radiol. 2009;20(8):10591065 .
21. Mortelé KJ, Segatto E, Ros PR. The infected liver: radiologic-pathologic correlation. Radiographics. 2004;24(4):937-955.

22. Lo JZ, Leow JJ, Ng PL, et al. Predictors of therapy failure in a series of 741 adult pyogenic liver abscesses. $J$ Hepatobiliary Pancreat Sci. 2015;22(2):156-165.

23. Alkofer B, Dufay C, Parienti JJ, et al.Are pyogenic liver abscesses still a surgical concern? A Western experience. HPB Surg. 2012;316013.

24. Cai YL, Xiong XZ, Lu J, et al. Percutaneous needle aspiration versus catheter drainage in the management of liver abscess: a systematic review and meta-analysis. HPB. 2015;17(3):195-201.

25. Kanda Y. Investigation of the freely available easy-to-use software 'EZR' for medical statistics. Bone Marrow Transplant. 2013;48(3):452-458.

26. Siu LK, Yeh KM, Lin JC, et al. Klebsiella pneumoniae liver abscess: a new invasive syndrome. Lancet Infect Dis. 2012;12(11):881-887.

27. Lee NK, Kim S, Lee JW, et al. CT differentiation of pyogenic liver abscesses caused by Klebsiella pneumoniae vs non-Klebsiella pneumoniae. Br J Radiol. 2001;84(1002):518-525.

28. Czerwonko ME, Huespe P, Bertone S, et al. Pyogenic liver abscess: current status and predictive factors for recurrence and mortality of first episodes. HPB. 2016;18(12):1023-1030.

29. Du ZQ, Zhang LN, Lu Q, et al. Clinical Characteristics and Outcome of Pyogenic Liver Abscess with Different Size: 15-Year Experience from a Single Center. Sci Rep. 2016;6:35890.

30. Ke S, Ding XM, Gao J, et al. Feasibility of radiofrequency ablation as an alternative to surgical intervention in patients with huge multiloculated pyogenic liver abscesses; A retrospective cohort study. Medicine. 2016;95(49): e5472.

31. Kusumoto K, Hamada A, Kusaka T, et al. A patient with sepsis and a gas-forming liver abscess caused by Clostridium perfringens treated with continuous perfusion drainage. Nihon Shokakibyo Gakkai Zasshi. 2014;111(7): 1416-1423.

32. Qian Y, Wong CC, Lai S, et al. A retrospective study of pyogenic liver abscess focusing on Klebsiella pneumoniae as a primary pathogen in China from 1994 to 2015. Sci Rep. 2016;6:38587.

33. Kong H, Yu F, Zhang W, et al. Clinical and microbiological characteristics of pyogenic liver abscess in a tertiary hospital in East China. Medicine (Baltimore). 2017;96(37):e8050.

34. Peris J, Bellot P, Roig P, et al. Clinical and epidemiological characteristics of pyogenic liver abscess in people 65 years or older versus people under 65: a retrospective study. BMC Geriatr. 2017;17(1):161. 